\title{
References
}

1 Morice AH, Millqvist E, Belvisi MG, et al. Expert opinion on the cough hypersensitivity syndrome in respiratory medicine. Eur Respir J 2014; 44: 1132-1148.

2 Pullerits T, Ternesten-Hasséus E, Johansson EL, et al. Capsaicin cough threshold test in diagnostics. Respir Med 2014; 108: 1371-1376.

3 Faruqi S, Thompson R, Wright C, et al. Quantifying chronic cough: objective versus subjective measurements. Respirology 2011; 16: 314-320.

4 Abdulqawi R, Dockry R, Holt K, et al. P2X3 receptor antagonist (AF-219) in refractory chronic cough: a randomised, double-blind, placebo-controlled phase 2 study. Lancet 2014 [In press DOI: 10.1016/S0140-6736(14) 61255-1].

5 Vertigan AE, Theodoros DG, Gibson PG, et al. Efficacy of speech pathology management for chronic cough: a randomised placebo controlled trial of treatment efficacy. Thorax 2006; 61: 1065-1069.

6 Ryan NM, Birring SS, Gibson PG. Gabapentin for refractory chronic cough: a randomised, double-blind, placebo-controlled trial. Lancet 2012; 380: 1583-1589.

\section{Screening for latent tuberculosis before tumour necrosis factor antagonist therapy}

To the Editor:

We read with great interest the recent letter by Woodfield et al. [1]. The authors report a retrospective study evaluating the use of either an interferon- $\gamma$ release assay (IGRA) result or a chest radiograph, alone, as a screening approach for identifying patients with latent tuberculosis (TB) infection (LTBI) before initiating tumour necrosis factor (TNF)- $\alpha$ antagonist therapy. In this study, 353 IGRA-negative patients were commenced on TNF- $\alpha$ antagonists for a variety of inflammatory conditions without further LTBI assessment; one patient subsequently developed active TB.

This approach differs from our practice, we currently advocate a "triple testing" approach in all patients referred for LTBI screening with a combination of risk stratification according to the British Thoracic Society (BTS) guidelines [2], tuberculin skin test (TST) and IGRA (T-Spot.TB: Oxford Immunotec, Oxford, UK) to aim for maximum sensitivity. We have previously published an evaluation of this approach in 137 patients receiving immunosuppression and found that $111(81.0 \%)$ were IGRA negative [3]. Of these, a total of 41 patients had either TST positive and/or were classified as high risk according to the BTS algorithm [3]. Therefore, use of the "IGRA alone" screening method, as advocated by WooDFIELD et al. [1], would have resulted in a sizeable reduction $(62 \%)$ in the total number of patients receiving chemoprophylaxis in our cohort.

A significant advantage of IGRAs is an improved specificity, but a reduced sensitivity in the context of immunosuppression is well recognised, particularly in those with HIV infection [4]. There are also descriptions of patients who have developed active TB despite a negative IGRA [5]. In addition, discrepancies have been illustrated between the two commercially available IGRAs [6].

Studies have shown that even in patients with prior bacillus Calmette-Guérin vaccine (BCG), a TST may add a further yield of those with possible LTBI [7]. Importantly, treatment on the basis of a positive TST and/or chest radiograph reduced the risk of TB reactivation in a high-risk population by $74 \%$, with low rates of isoniazid-induced hepatotoxicity [8].

In the data, detailed by Woodfield et al. [1], a further 41 patients in the IGRA-negative group, with available demographic data, could have been offered chemoprophylaxis on the basis of risk stratification. The authors note this would be associated with additional resource costs. Use of the IGRA-alone screening approach in their cohort led to one patient developing active TB. The authors do not disclose the ethnicity of this case or elaborate on prior immunosuppressant therapy used, but do comment that the patient would have otherwise been treated based on risk stratification. 
In absolute terms this represents a small number of cases; however, on a population level, this may equate to a significant number of new cases of $\mathrm{TB}$ that could otherwise have been prevented with chemoprophylaxis. Extrapolated, the incidence of TB reactivation in this study (one in 488) would equate to an estimated incidence of 205 per 100000 , a rate comparable to high-risk ethnic groups.

Costs incurred for a typical case of TB are estimated at $£ 5000$ for treatment of the index case [9], but there are additional costs associated with case finding and screening of close contacts. Furthermore, the potential negative impact on public health due to additional cases of $\mathrm{TB}$, with the propensity for spread of disease within a population, should be considered. Crucially, fatalities have been recorded in cases of TB following initiation of TNF- $\alpha$ antagonist therapy where there is a significant over-representation of disseminated disease [10].

There may be a substantial future risk of TB reactivation in those 41 "high-risk", IGRA-negative patients that was not captured by this study's median 28.8 month follow-up. Cases of TB have been diagnosed up to 108 months after starting TNF- $\alpha$ antagonist therapy, with a median of 89 months for patients receiving etanercept [11]. Considering over a third of the cohort studied by WoodFIELD et al. [1] were taking etanercept, a longer follow-up period is required before definitive comment can be made on the benefits of this screening method.

We recognise that false-positive results are likely to be higher with a triple testing strategy and WooDFIELD et al. [1] suggest this approach may result in unnecessary treatment and exposure to a risk of therapy-induced hepatotoxicity. Only two (3.1\%) patients in our study developed treatment-limiting hepatotoxicity [3].

Neither the TST nor IGRA appear able to fully predict who will progress from latent to active TB. There is a demand for longitudinal studies assessing the risk of progression to TB in TST- versus IGRA-based strategies. Screening recommendations to identify patients with LTBI vary throughout Europe [12]. The World Health Organization guidelines currently recommend the use of either IGRA or TST to test for LTBI in high-risk populations, including those initiating an anti-TNF therapy [13].

Prevention of TB transmission with LTBI treatment strategies to protect high-risk groups remains an important strategy to reduce the prevalence and mortality of TB [12]. In a recently published survey of TB elimination activities in Europe, 22 countries reported LTBI screening in high-risk groups such as patients due to commence anti-TNF therapy [14]. Only six (19.4\%) countries were able to provide data on rates of LTBI preventative treatment, making it difficult to evaluate the overall public health impact of treatment strategies.

The debate over the optimal screening strategy for patients commencing TNF- $\alpha$ antagonists will continue. One potential advantage of using IGRA testing over TST may be a reduction of false-positive and false-negative results in a sub-group of patients treated with corticosteroid therapy and/or with a history previous BCG vaccination [15]. It remains unclear whether a pragmatic, and potentially cost-effective, "IGRA-only" strategy is preferable to a more intensive, but potentially more sensitive, "triple test" approach. Ultimately, larger studies with prospective longitudinal data of each approach are required. It is also important to evaluate each approach with a robust cost-effectiveness analysis and we hope the latest revision of the UK National Institute for Health and Care Excellence guidelines, due in 2015, may help answer this question. Until such data is available and in the absence of a sufficiently sensitive single biomarker or test, we would not advocate abandoning a more sensitive approach based on triple testing, especially in areas where the local incidence of TB may be high (even in the setting of a low incidence country).

@ERSpublications

Reliance on interferon- $\gamma$ release assays alone for screening before anti-TNF therapy may miss patients with latent TB http://ow.ly/JXx74

Richard J. Hewitt ${ }^{1}$, Aran Singanayagam ${ }^{1}$, Saranya Sridhar ${ }^{2}$, Melissa Wickremasinghe ${ }^{1,2}$ and Onn Min Kon ${ }^{1,2}$

${ }^{1}$ Chest and Allergy Dept, St Mary's Hospital, Imperial College NHS Trust, London, UK. ${ }^{2}$ Imperial College London, London, UK.

Correspondence: Onn Min Kon, Imperial College London, St. Mary’s Campus, Norfolk Place, London, W2 1PG, UK. E-mail: onn.kon@imperial.nhs.uk

Received: Oct 022014 | Accepted after revision: Jan 202015

Conflict of interest: Disclosures can be found alongside the online version of this article at erj.ersjournals.com

\section{References}

1 Woodfield G, Passey-Heaton B, Chakrabarti A, et al. An evaluation of the use of a negative interferon- $\gamma$ release assay for tuberculosis screening before TNF antagonist therapy. Eur Respir J 2014; 44: 1369-1372.

2 British Thoracic Society Standards of Care Committee. BTS recommendations for assessing risk and for managing Mycobacterium tuberculosis infection and disease in patients due to start anti-TNF- $\alpha$ treatment. Thorax 2005; 60: 800-805. 
Singanayagam A, Manalan K, Sridhar S, et al. Evaluation of screening methods for identification of patients with chronic rheumatological disease requiring tuberculosis chemoprophylaxis prior to commencement of TNF- $\alpha$ antagonist therapy. Thorax 2013; 68: 955-961.

4 Cattamanchi A, Smith R, Steingart KR, et al. Interferon-gamma release assays for the diagnosis of latent tuberculosis infection in HIV-infected individuals: a systematic review and meta-analysis. J Acquir Immune Defic Syndr 2011; 56: 230-238.

5 Chen DY, Shen GH, Chen YM, et al. Biphasic emergence of active tuberculosis in rheumatoid arthritis patients receiving TNF $\alpha$ inhibitors: the utility of IFN $\gamma$ assay. Ann Rheum Dis 2012; 71: 231-237.

6 Mariette X, Baron G, Tubach F, et al. influence of replacing tuberculin skin test with ex vivo interferon $\gamma$ release assays on decision to administer prophylactic antituberculosis antibiotics before anti-TNF therapy. Ann Rheum Dis 2012; 71: 1783-1790.

7 Kleinert S, Tony H, Krueger K, et al. Screening for latent tuberculosis infection: performance of tuberculin skin test and interferon- $\gamma$ release assays under real-life conditions. Ann Rheum Dis 2012; 71: 1791-1795.

8 Carmona L, Gómez-Reino JJ, Rodríguez-Valverde V, et al. Effectiveness of recommendations to prevent reactivation of latent tuberculosis infection in patients treated with tumor necrosis factor antagonists. Arthritis Rheum 2005; 52: 1766-1772.

9 The ongoing problem of tuberculosis in the UK. Lancet 2013; 381: 1431.

10 Dixon WG, Hyrich KL, Watson KD, et al. Drug-specific risk of tuberculosis in patients with rheumatoid arthritis treated with anti-TNF therapy: results from the British Society for Rheumatology Biologics Register (BSRBR). Ann Rheum Dis 2010; 69: 522-528.

11 Abreu C, Magro F, Santos-Antunes J, et al. Tuberculosis in anti-TNF- $\alpha$ treated patients remains a problem in countries with an intermediate incidence: analysis of 25 patients matched with a control population. J Crohns Colitis 2013; 7: e486-e492.

12 Diel R, Loddenkemper R, Zellweger JP, et al. Old ideas to innovate tuberculosis control: preventive treatment to achieve elimination. Eur Respir J 2013; 42: 785-801.

13 World Health Organization. Guidelines on the Management of Latent Tuberculosis Infection. Geneva, World Health Organization, 2015. Available from: www.who.int/tb/publications/ltbi_document_page/en/

14 D'Ambrosio L, Dara M, Tadolini M, et al. Tuberculosis elimination: theory and practice in Europe. Eur Respir J 2014; 43: 1410-1420.

15 Ruan Q, Zhang S, Ai J, et al. Screening of latent tuberculosis infection by interferon- $\gamma$ release assays in rheumatic patients: a systemic review and meta-analysis. Clin Rheumatol 2014 [In press DOI: 10.1007/s10067-014-2817-6].

Eur Respir J 2015; 45: 1510-1512 | DOI: 10.1183/09031936.00194314 | Copyright @ERS 2015

From the authors:

We thank Richard Hewitt and colleagues for their comments. Determining the best strategy and combination of tests for detection of latent tuberculosis (TB) infection (LTBI) is limited by the lack of gold standard for the diagnosis of LTBI. Increasing the number of tests may improve the sensitivity of latent TB detection, but at the cost of specificity, with an increased false-positive rate.

Hewitt and colleagues use the active TB rate (1/488) in our cohort [1], to justify a more comprehensive latent TB detection strategy than interferon- $\gamma$ release assay (IGRA) screening. However, there is no evidence provided that suggests an alternative regimen would lead to a lower active-TB rate in tumour necrosis factor- $\alpha$ antagonist (TNF- $\alpha$ ) treated patients. In fact, higher TB rates have been described in similar cohorts despite more intensive screening (i.e. use of tuberculin skin test (TST) and IGRA), or even following LTBI treatment $[2,3]$, which is known to only be $60-90 \%$ effective [4]. In addition, other groups that have similarly used single IGRA testing, in this context, have not demonstrated patients go on and develop active TB $[5,6]$.

Hewitt and colleagues go on to suggest that there may be a substantial future risk of TB activity beyond the median 28.8 day follow-up in our study. As described in our article, most studies demonstrate TB activation before this time. The authors cite a study to describe a longer latency period [2]; however they just report data based on three etanercept patients (with median times of 6-7 months for diagnosis of active TB for the other 22 TNF- $\alpha$ patients in that study). Such a long latency period is most likely to reflect new TB infection, rather than reactivation, and initial LTBI treatment would not affect this. Indeed this point is made in the article they cite and by the authors themselves in a further article describing the need for repeated longitudinal LTBI testing [7].

The addition of further testing, as suggested with the triple strategy of TST, IGRA and risk tables, will increase the number of false positives and the accompanying unnecessary use of latent TB treatment in a number of patients. They report a hepatotoxicity rate sufficient to limit treatment in $3.1 \%$ of patients [8]. In that study, 41 out of 111 IGRA-negative patients were treated for LTBI, which would equate to an extra 1.27 patients being expected to develop significant hepatotoxicity. Using similar extrapolation methods to the authors, this would equate to a hepatotoxicity rate of 1145 per 100000 in the IGRA-negative population, which is significantly higher than the TB reactivation risk (based on our study) in this group. 